\title{
Effect of cropping system and age of plant at harvest on tuber rot and performance of elite cassava varieties in derived savannah
}

\author{
ODEDINA $^{1}$ J. N., ADIGBO ${ }^{2 *}$ S. O., KULAKO ${ }^{3}$ P., ILUEBBEY $^{3}$ P., FABUNMI ${ }^{1}$ T. O., AFOLABI ${ }^{4}$ C. G., and \\ OSIPITAN $^{4}$ A. A.
}

Received February 12, 2016; accepted August 03, 2017.

Delo je prispelo 12. februarja 2016, sprejeto 03. avgusta 2017.

\begin{abstract}
Devastated tuber rot disease among farmers prompted the evaluation of the elite improved varieties in the intercrop and the practice of delaying harvesting when there is glut in the market necessitated this study. Trial was carried out at the Federal University of Agriculture, Abeokuta between 2011 and 2014 to evaluate yield performance of 21 elite cassava varieties planted as sole crop verse intercropped and harvested at different age. The $2 \times 21 \times 3$ factorial experiment was laid out in randomized complete block design and replicated three times. The tuber yield obtained from sole plot in 2011/2012 cropping season was significantly higher than intercrop whereas those of 2012/2014 cropping season were similar. Land Equivalent Ratio was above one in both cropping seasons indicating that the performance of the improved varieties in intercrop was efficient. The pooled mean tuber yield showed that TMS 30572, 92/0326, 95/0211, 01/1371, 00/0338, 01/0046, 00/0098, 01/1097, 01/0085, 98/0581 and $98 / 510$ were among the top eight varieties. Harvesting could be delayed up to 15 months after planting to reduce tuber rot.
\end{abstract}

Key words: cassava; intercrop; tuber rot; delay harvest; Nigeria
IZVLEČEK

\section{UČINKI NAČINA GOJENJA IN STAROSTI RASTLIN OB SPRAVILU NA POJAVLJANJE GNILOBE GOMOLJEV PRI ELITNIH SORTAH MANIOKE V ANTROPOGENI SAVANI NIGERIJE}

Zaradi prakticiranja odloga spravila pridelka manioke, kadar se pojavlja njen višek na trgu in pojavljanja uničujoče gnilobe gomoljev, se je pojavila potreba po ovrednotenju elitnih sort te tropske gomoljevke, gojene v medsadnji. Poskus je bil izveden na Federal University of Agriculture, Abeokuta med 2011 in $2014 \mathrm{z}$ namenom ovrednotenja pridelka 21 elitnih sort manioke, posajene $\mathrm{v}$ monokulturi ali $\mathrm{v}$ kombinaciji $\mathrm{z}$ drugimi kulturami in pospravljene $\mathrm{v}$ različnih časovnih obdobjih. Faktorski $2 \times 21 \times 3$ poskus je bil izveden po sistemu naključnih blokov s tremi ponovitvami. Pridelek gomoljev na površinah $\mathrm{z}$ monokulturo je bil v rastni sezoni 2011/2012 značilno večji kot na površinah $\mathrm{z}$ medsadnjo, $\mathrm{v}$ rastni sezoni 2012/2014 pa sta bila pridelka podobna. Ekvivalent zemljišča je bil nad ena $v$ obeh rastnih sezonah in kaže prednost izboljšanih sort, gojenih $\mathrm{v}$ medsadnji. Analiza povprečnih vrednosti pridelka gomoljev je pokazala, da so bile sorte TMS 30572, 92/0326, 95/0211, 01/1371, 00/0338, 01/0046, 00/0098, 01/1097, 01/0085, 98/0581 in 98/510 med osmimi najdonosnejšimi. Za zmanjšanje gnilobe je izkop gomoljev lahko zamaknjen do 15 mesecev po sadnji.

Ključne besede: manioka; medkultura; gniloba gomoljev; poznejši izkop; Nigeria

\section{INTRODUCTION}

Cassava (Manihot spp.) belongs to the family of Euphorbiaceae. Cassava is one of the most important food crops in Africa, South America and Asia. It derives its importance from the fact that its starchy, thickened, tuberous roots are a valuable source of cheap calories, especially in the developing countries where calorie deficiency and malnutrition are widely spread. Its usage as a source of ethanol for fuel, energy in animal feed,

\footnotetext{
1 Federal University of Agriculture, Abeokuta, Nigeria; Plant Physiology and Crop Production Department

2 Federal University of Agriculture, Abeokuta, Nigeria; Crop Research Programme, IFSERAR; *Corresponding author: adigboso@ funaab.edu.ng

3 International Institute of Tropical Agriculture, Ibadan, Nigeria

4 Federal University of Agriculture, Abeokuta, Nigeria; Crop Protection Department
} 


\section{J. N. ODEDINA et al.}

and starch for industries is increasing. Cassava contributes the largest share of daily per capita food consumption (1.6 kg) in Nigeria (FAOSTAT, 2003) and ranked number one among the top 20 commodities produced in Nigeria (www.fao.org/faostat/en/\#rankings/commodities_by_co untries) for more than estimated 800 million people around the world (Akparobi et al., 1998; Lebot, 2009).

Nigeria, Thailand, Indonesia and Brazil were ranked as first, second, third and fourth respectively, among the top 20 countries producing cassava in the world. The current estimated cassava production in 2013 for Nigeria, Thailand, Indonesia and Brazil were 47.4, 30.2, 23.0 and 21.5million tonnes, respectively (www.fao.org/faostat/en/\#rankings/countries_by_comm odities). Total area harvested in 2009 was 3.13 million ha, with an average yield of $11.7 \mathrm{t} \mathrm{ha}^{-1}$ (FAO, 2010). It is produced predominantly $(99 \%)$ by small farmers with 1-5 ha of land intercropped with yams, maize, or legumes in the rainforest and savannah agro-ecologies of Southern, Central, and lately Northern Nigeria. The world production of cassava root was estimated to be 184 million tonnes in 2002.

IFSERAR, (2009) conducted a diagnostic survey in South West Nigeria and reported that the local varieties grown among the farmers were not only low yielders but their ability to tolerate, or resist new strains of diseases and pests occasioned, perhaps, by climate change. Mwangi et al. (2004) similarly reported that the root rots are an important constraint to cassava production in humid forest and forest transition of Central and West Africa and can impact negatively on food security to several millions people inhabiting the regions. Rotting is known to increase significantly if mature plants are left in the soil for extended period of time (Oyeka, 2004). Yield loss was estimated at 20 to $100 \%$ in Democratic Republic of Congo (Mwangi et al., 2004). These challenges necessitated the evaluation of 21 promising varieties collected from International Institute of Tropical Agriculture (IITA) in maize/cassava intercrop.
IITA have released several high yielding varieties but there performances in the intercrop as well as their tolerant or resistant level to cassava root rot disease have not been documented. There is therefore the need to ascertain the performance of these elite cassava varieties under the predominant intercropping systems among the resource constraint farmers in the region. Besides, the highly perishable nature of cassava tubers has compelled the farmers to harvest only when there is availability of market or family need. This delay harvesting enables farmers to leave the mature plant in the soil as a form of storage. This storage period enable the farmers to keep the fresh tuber in good quality for an extended period. Growth and dry matter accumulated will continue since cassava is believed to mature 7-24 months. Most cassava varieties attain optimum weight at 18 months after planting when starch accumulation is highest (Ekanayake et al., 1997). Hammer et al. (1987), who evaluated sequential harvests to age 24 months, reported that root rot occurred in the second year. Sagrilo et al. (2006) quoted Sagrilo et al. (2002) that cassava harvested at 21 months could improve storage root yield compared to 12 months. Ebah-Djedji et al.(2012) who harvested cassava sequentially at 11, 13, 15 and 17 months after planting in Cote d' Ivoire recommended that tuberous root of improved cassava varieties should be harvested at 13 MAP to obtain optimum dry matter content.

These inconsistencies in the appropriate time of harvesting to obtain optimum dry matter content and quality is further aggravated by the prevailing tuber rot. Consequently, there is the need to ascertain the appropriate time to harvest these elite cassava varieties. This will ensure maximum dry matter accumulation without losing much of the tuber to root rot, particularly when harvesting is delayed because of poor market arrangement. The objectives of this study therefore were to: evaluate the performance of the improved varieties, 2) investigate the effect of intercropping on the elite cassava varieties and 3) determine the effect of delay harvest on the cassava tuber rot.

\section{MATERIALS AND METHODS}

The study was carried out at the Institute of Food Security, Environmental Resource and Agricultural Research (IFSERAR) farm, Federal University of Agriculture, Abeokuta in 2011/2012 and repeated in 2012-2014 cropping seasons. The experiment was laid out in a Randomized Complete Block Design in split plot and replicated three times in the 2011/2012. Cropping systems and variety factors were assigned to the main plot and sub plot, respectively. However, in 2012-2014 cropping season, harvesting date (12, 15 and
18 months) was varied as the third factor i.e. sub subplot (split split-plot) to gain additional information. The intercrop proportion mixture and population adopted was additive series. Table 1 shows the twenty one elite cassava varieties collected from International Institute of Tropical Agriculture (IITA), Ibadan. Benlate treated stem cuttings of $25 \mathrm{~cm}$ were planted into plot size of 9 $\mathrm{m} \times 7 \mathrm{~m}$ (1.2 ha experimental field) at spacing of $1 \mathrm{~m} \mathrm{x}$ $1 \mathrm{~m}$ in July 2011 and harvested in July, 2012. The cuttings obtained from the harvest were replanted in 
July 2012 and harvested sequentially in July (12 months after planting MAP), October 2013 (15 MAP) and January 2014 (18 MAP). Three seeds per hole of treated maize 'SUWAN 1' variety was alternately planted inbetween cassava stands (in the intercrop plots only) to evaluate the performance of cassava under intercropping (i.e. additive series). Maize was harvested at green stage. Weeding was carried out at 3,9 12 WAP. Other weedings were done once in a month. Fertilizer 400 $\mathrm{kg} / \mathrm{ha} \mathrm{N}: \mathrm{P}: \mathrm{K}: \mathrm{Mg}(12: 12: 17: 2)$ was applied in the 2011/2012 cropping season whereas 2012/2014 trial did not receive fertilizer because of circumstance beyond our control.

\subsection{Data collection on cassava}

\subsubsection{Plant height $(\mathrm{cm})$ :}

5 randomly selected cassava plants within the plot were measured with aid of graduated meter rule from the ground level to the highest leaf.

\subsubsection{Stem girth $(\mathrm{mm})$ :}

Vernier caliper was used to determine the stem girth (at $10 \mathrm{~cm}$ above the ground) of 5 randomly selected cassava stems within the plot.

Table 1: Selected cassava varieties used for the experiment

\begin{tabular}{|l|l|}
\hline Variety & Tuber color \\
\hline TMS98/0581 & White \\
\hline TMS 01/1797 & White \\
\hline TMS 95/0211 & White \\
\hline TME 1 & White \\
\hline TMEB 693 & White \\
\hline TMS 01/0046 & White \\
\hline TMS 01/0093 & White \\
\hline TMS 00/0338 & White \\
\hline TMS 01/1097 & White \\
\hline TMS 01/1086 & White \\
\hline TME B 419 & White \\
\hline TMS 30572 & White \\
\hline TMS 01/1371 & Yellow \\
\hline TMS 01/0085 & White \\
\hline TMS 98/0510 & White \\
\hline TMS 01/0131 & White \\
\hline TMS 98/0505 & White \\
\hline TMS 92/0326 & White \\
\hline TMS 01/0098 & White \\
\hline TMS 01/1368 & Yellow \\
\hline TMS 97/JW2 & Yellow \\
\hline
\end{tabular}




\section{RESULTS AND DISCUSSION}

\subsection{Influence of intercropping on the plant height of elites cassava varieties at 12 MAP}

Plant height and stem girth are essential component to determine plant growth particularly when intercrop is involved. The plant height of the 21 varieties obtained at 12 months after planting varied significantly $(\mathrm{P}>0.05)$ from each other in the 2011/2012 cropping season (Table 2). TME B 419 had the tallest plants but comparable to TMEB 693, TMS 01/1097, TMS 01/1797, TME 1, TMS 01/1086, TMS 01/1371,TMS 97/JW2 and TMS 92/0326. Whereas, TMS 98/505 had the shortest plant which was similar to those of TMS 01/0098, TMS 01/0131, TMS 01/0046, TMS 01/0093 and TMS 00/0338. In 2012/2014 cropping season, TMS 97/JW2 and TMS01/0093 had the tallest plants at 12 MAP but were similar to those of TMS 95/0211, TME 1, TMS 00/00338, TME B 419, TMS 30572, TMS 01/1371, TMS 01/0085, TMS 98/0510, TMS 92/0326, TMS 01/0098 and TMS 01/1386 (Table 3). However, TMS 01/1797, TMEB 693, TMS 01/0046, TMS 01/1097, TMS 01/1086, TMS 01/0131 and TMS $98 / 0505$ had the shortest plants in 2012/2014. The plant heights in the two seasons were at variance except those of TMS 97/JW2, TME B 419 and TMS 01/1371 which were consistently top on the list, whereas TMS 01/0131, TMS 01/0046 and TMS 98/0505 constantly had the shortest plants. This consistency in plant height implies that the varieties were stable in the different environment, whereas the others were influenced more by the environment.

\subsection{Influence of intercropping on the tuber number of elites cassava varieties at 12 MAP}

The number of fresh tubers observed in 2011/2012 on cassava varieties TME 1, TMS 98/0505, TMS 97/JW2, TMS 98/0581, TMS 01/1097, TMS 01/1386, TMS 30572, TMS 01/1086, TMS 01/0085 and TMS 00/0338 were similar but significantly higher than those of TMS 01/1371 and TMS 01/0131 varieties in 2011/2012 cropping season (Table 2). The varieties TMS 30572, TME 1, TMS 01/0093, TMS 00/0338, TMS 1097, TMS 01/0046, TMEB 693, TMS 98/0510 and TMS 92/0326 in 2012/2014 were among the top varieties with high number of fresh tuber while TME B 419 had the least (Table 3). TME 1, TMS 01/1097, TMS 30572 and TMS $00 / 0338$ were constantly ranked amongst the top varieties with high number of tubers in 12 MAP of the two seasons. The variance in tuber number could be genetically inherent and was considered as vital yield attribute that contributes immensely to the increase in tuber yield. It has been documented that the increase in yield were attributed to increase in number of tuber/stand and single root mass (Kogram et al., 2002).

\subsection{Influence of intercropping on the tuber girth of elites cassava varieties at 12 MAP}

The cropping system and variety did not influence tuber girth in 2011/2012 (Table 2), however, in 2012/2014 the varieties varied significantly among each other in 2012/2014 at 12 MAP (Table 3). 'TMS 98/0510' had the highest tuber girth while 'TMS 97/JW2' had the least. The stem girths of the varieties were influenced by cropping systems in both seasons of the trial at 12 MAP (Tables 2 and 4). However, the varieties TMS 00/0338 and TMS 98/0505 consistently recorded the highest and the lowest, respectively in 12 MAP of both cropping season.

\subsection{Influence of intercropping on the root rot of elites cassava varieties at 12 MAP}

The cropping systems did not influence tuber rot infection in both seasons but there were significant differences among the varieties in 12 MAP of 2011/2012 (Table 2), they were however similar in 2012/2014 (Table 4). The rot incidence observed in 2011/2012 was high and ranges between 9.8 and $22.5 \%$ while that of 2012/2014 was low and range between 0.00 and $0.94 \%$ at $12 \mathrm{MAP}$. The lost incurred during 2011/2012 cropping season is in consonant with the finding of Mwangi et al. (2004) who documented 20 to $100 \%$ tuber lost.

\subsection{Influence of intercropping on the LER and tuber fresh mass of elites cassava varieties at 12 MAP}

The Land Equivalent Ratio (LER) was similar in both cropping season but above one suggesting that intercrop plots was more productive. The fresh tuber mass of the varieties varied in the two cropping seasons at 12 MAP (Tables 2 and 4). Sole cassava plots had significantly higher tuber mass than intercrop in 2011/2012 (Table 2) but similar 2012/2014 cropping seasons (Table 4). In 2011/2012 cropping season, TMS 98/0505, TMS 97/JW2, TME 1, TMS 30572, TMS 95/0211, TMS 92/0326, TMS 01/0085, TMS 01/0098, and TMS 98/0581 varieties were the nine topmost in terms of fresh tuber mass at 12 MAP. Whereas the following varieties: TMS 01/1086, TMS 01/1368, TMS 98/0510, TMS 01/1097, TMS 01/1371, TMS 01/1797 and TMS 01/0046 closely followed. However, 'TMEB 693' had the lowest tuber yield. The fresh tuber yields range between 27.5 and $57.4 \mathrm{t} \mathrm{ha}^{-1}$ in 2011/2012 while those of 2012/2014 was 12 to $32.3 \mathrm{t} \mathrm{ha}^{-1}$. The yield range obtained in 2011/2012 was substantially higher than that of 2012/2014 at $12 \mathrm{MAP}$ as expected. However, the results obtained in 2012/2014 was comparable to range of 9.9 to $30.1,8.49$ to 28.38 , and 10.0 to $26.9 \mathrm{t} \mathrm{ha}^{-1}$ as 
reported by IITA (1987), Maroya et al. (2010) and Ssemakula and Dixon (2007), respectively.

The eleven topmost varieties in 2012/2014 cropping season at 12 MAP, were TMS 00/0338, TMS 30572, TMS 92/00326, TMS 01/1097, TMS 98/0581, TMS 01/0046, TME 1, TMS 98/0510, TMS 01/0085, TMS $01 / 1371$, and TMS $95 / 0211$ in that order. These were closely followed by TMS 01/1086, TMS 01/0093, TMS 01/1797, TMS 01/0098, TMS 01/1368, TME B 419 and TMS 01/0131 varieties. While TMS 97/JW2 variety had the lowest tuber yield. The variation in tuber yield of the varieties agreed with the finding of Howeler (2007); Mulualem and Ayenew (2012); Odedina et al. (2012) who reported that yields of cassava roots vary with cultivars, plant growth conditions (soil, climate, rainfall) and agronomic practices. It is pertinent to note that the variation in tuber yield of the 21 varieties was only observed in 12 MAP (Tables 2 and 4) while the harvest made at 15 and 18 MAP had similar tuber yield. This is, perhaps, an indication that maturity had not been attained and thus dry matter accumulation were at variance at 12 MAP. Although, number of tuber, stem girth and tuber girth varies among the varieties at 15 and 18 MAP (Table 3) but all the varieties had similar tuber yield (Table 4). This is an indicative of the fact that all the varieties tested in this trial attained maturity period after 12 MAP. Based on the definition of maturity period of cassava by Benesi et al. (2008) is the point where maximum or near maximum yield is obtained.

The topmost 3 consistent varieties in the two cropping seasons were TMS 30572, TMS 92/0326 and TMS 98/0581. Although 'TMS 01/1371' and 'TMS 01/1386' were not listed among top yielder, but had beta carotene as an advantage and statistically comparative yield with the top varieties in the two cropping seasons.

Table 2: Influence of intercropping on the tuber rot, agronomic parameters and tuber yield performance of elites cassava varieties in 2011/2012 cropping season

\begin{tabular}{|c|c|c|c|c|c|c|c|}
\hline Treatment & $\begin{array}{l}\text { Plant height } \\
\text { (m) }\end{array}$ & $\begin{array}{l}\text { Fresh } \\
\text { tuber } \\
\text { no.plant }^{-1} \\
\end{array}$ & $\begin{array}{l}\text { Tuber girth } \\
(\mathrm{mm})\end{array}$ & $\begin{array}{l}\text { Stem girth } \\
(\mathrm{mm})\end{array}$ & $\begin{array}{l}\text { Rot incidence } \\
(\%)\end{array}$ & LER & $\begin{array}{l}\text { Fresh tuber } \\
\text { mass }\left(\mathrm{t} \mathrm{ha}^{-1}\right)\end{array}$ \\
\hline \multicolumn{8}{|l|}{ Cropping System (CS) } \\
\hline Sole & 2.74 & 7.2 & 63.66 & 28.93 & 14.3 & - & $44.2 \mathrm{a}$ \\
\hline Intercrop & 2.72 & 6.9 & 62.22 & 28.52 & 14.3 & - & $39.4 b$ \\
\hline LSD & NS & NS & NS & NS & $\mathrm{NS}$ & - & 2.07 \\
\hline \multicolumn{8}{|l|}{ Variety (V) } \\
\hline TMS 98/0581 & $2.78 \mathrm{bcde}$ & 7.3a-e & 69.13 & $32.75 \mathrm{ab}$ & $22.5 \mathrm{a}$ & 1.96 & 41.7abc \\
\hline TMS 01/1797 & $2.98 \mathrm{abc}$ & 7.0b-f & 59.97 & $27.94 \mathrm{abc}$ & $18.3 \mathrm{a}-\mathrm{d}$ & 1.73 & $38.5 \mathrm{bcd}$ \\
\hline TMS 95/0211 & 2.61cdef & $6.8 b-f$ & 67.04 & $28.75 \mathrm{abc}$ & 11.7ef & 1.40 & $46.5 \mathrm{abc}$ \\
\hline TME 1 & 2.93abcd & $8.7 \mathrm{a}$ & 64.40 & $28.26 \mathrm{abc}$ & $10.2 \mathrm{f}$ & 1.96 & $47.6 \mathrm{abc}$ \\
\hline TMEB 693 & $3.13 \mathrm{ab}$ & 7.0b-f & 57.22 & $27.12 \mathrm{abc}$ & $13.4 d-f$ & 1.56 & $27.5 \mathrm{~d}$ \\
\hline TMS 01/0046 & 2.49efg & $6.7 c-f$ & 66.14 & $32.22 \mathrm{abc}$ & $16.3 b-f$ & 1.86 & $38.1 \mathrm{bcd}$ \\
\hline TMS 01/0093 & 2.49efg & $6.2 \mathrm{~d}-\mathrm{f}$ & 60.44 & $25.76 b c$ & $18.2 \mathrm{a}-\mathrm{d}$ & 1.60 & $35.3 \mathrm{~cd}$ \\
\hline TMS 00/0338 & $2.31 \mathrm{fg}$ & 7.5a-e & 58.90 & $34.24 \mathrm{a}$ & 12.0ef & 1.56 & $34.5 \mathrm{~cd}$ \\
\hline TMS 01/1097 & $3.00 \mathrm{abc}$ & 6.0ef & 61.23 & $29.72 \mathrm{abc}$ & $11.8 \mathrm{ef}$ & 1.73 & 40.1bcd \\
\hline TMS 01/1086 & 2.69adef & $8.0 \mathrm{abc}$ & 60.02 & $26.88 \mathrm{abc}$ & $13.5 c-f$ & 1.96 & $41.2 \mathrm{bc}$ \\
\hline TME B 419 & $3.23 \mathrm{a}$ & $6.7 c-f$ & 61.85 & $27.96 \mathrm{abc}$ & $12.9 \mathrm{~d}-\mathrm{f}$ & 1.73 & $37.0 \mathrm{~cd}$ \\
\hline TMS 30572 & $2.78 \mathrm{bcde}$ & $8.0 \mathrm{abc}$ & 65.23 & $28.68 \mathrm{abc}$ & $13.6 c-f$ & 1.73 & $47.2 \mathrm{abc}$ \\
\hline TMS 01/1371 & 2.90abcd & $5.7 f$ & 58.45 & 29.19abc & $20.1 \mathrm{ab}$ & 1.56 & $39.4 \mathrm{bcd}$ \\
\hline TMS 01/0085 & $2.75 \mathrm{cde}$ & 7.3a-e & 63.83 & $27.81 \mathrm{abc}$ & 16.6a-e & 1.50 & $45.8 \mathrm{abc}$ \\
\hline TMS 98/0510 & $2.66 \mathrm{cdef}$ & $6.2 \mathrm{~d}-\mathrm{f}$ & 73.30 & $31.51 \mathrm{abc}$ & $19.5 \mathrm{abc}$ & 2.03 & $40.6 \mathrm{bcd}$ \\
\hline TMS 01/0131 & 2.54defg & $5.7 f$ & 60.41 & $27.23 \mathrm{abc}$ & 16.6a-e & 1.56 & $36.6 \mathrm{~cd}$ \\
\hline TMS 98/0505 & $2.21 \mathrm{~g}$ & $8.2 \mathrm{ab}$ & 66.08 & $24.52 \mathrm{c}$ & $12.9 \mathrm{~d}-\mathrm{f}$ & 1.70 & $57.4 \mathrm{a}$ \\
\hline TMS 92/0326 & 2.85abcde & 7.0b-f & 68.70 & $28.65 \mathrm{abc}$ & $13.9 c-f$ & 1.50 & 45.9abc \\
\hline TMS 01/0098 & $2.40 \mathrm{fg}$ & $6.7 c-f$ & 61.75 & $28.95 \mathrm{abc}$ & $15.0 \mathrm{~b}-\mathrm{f}$ & 1.90 & $45.1 \mathrm{abc}$ \\
\hline TMS 01/1368 & $2.82 \mathrm{bcde}$ & 7.7abc & 61.01 & $31.16 a b c$ & $11.5 \mathrm{ef}$ & 1.80 & $40.3 \mathrm{bcd}$ \\
\hline TMS 97/JW2 & 2.85a-e & $8.2 \mathrm{ab}$ & 56.58 & $25.97 \mathrm{abc}$ & $9.8 \mathrm{f}$ & 1.90 & $51.3 \mathrm{ab}$ \\
\hline SE (V) & 0.201 & 0.75 & 12.53 & 4.147 & 3.07 & NS & 6.69 \\
\hline CS X V & NS & NS & $\mathrm{NS}$ & NS & $\mathrm{NS}$ & $\mathrm{NS}$ & $\mathrm{NS}$ \\
\hline
\end{tabular}

NS = not significant 


\section{J. N. ODEDINA et al.}

Table 3: Influence of intercropping on agronomic performance of elite cassava varieties at different age of plant in 2012/2014 cropping season

\begin{tabular}{|c|c|c|c|c|c|c|c|c|c|}
\hline \multirow[b]{2}{*}{ Treatment } & \multicolumn{2}{|c|}{ Plant height (m) } & \multirow[b]{2}{*}{18 MAP } & \multicolumn{3}{|c|}{ Fresh tuber No. } & \multicolumn{2}{|c|}{ Tuber girth (mm) } & \multirow[b]{2}{*}{18 MAP } \\
\hline & $12 \mathrm{MAP}$ & $15 \mathrm{MAP}$ & & $\begin{array}{l}12 \\
\text { MAP }\end{array}$ & $\begin{array}{l}15 \\
\text { MAP }\end{array}$ & $18 \mathrm{MAP}$ & $12 \mathrm{MAP}$ & 15 MAP & \\
\hline \multicolumn{10}{|c|}{ Cropping systems (CS) } \\
\hline Sole & 2.25 & 2.71 & 2.87 & 6.1 & 6.6 & 5.71 & 57.22 & 67.1 & 64.1 \\
\hline Intercrop & 2.31 & 2.76 & 2.94 & 6.3 & 6.6 & 5.67 & 58.66 & 66.9 & 65.0 \\
\hline LSD & NS & NS & NS & NS & NS & NS & NS & NS & NS \\
\hline \multicolumn{10}{|l|}{ Variety (V) } \\
\hline TMS 98/0581 & $2.66 \mathrm{ab}$ & $3.15 \mathrm{abc}$ & $3.04 a b c$ & $6.2 b-f$ & $6.7 \mathrm{a}-\mathrm{d}$ & 5.6a-e & 64.1ab & 66.1a-e & $69.5 \mathrm{abc}$ \\
\hline TMS 01/1797 & $1.70 \mathrm{~d}$ & $2.12 \mathrm{~d}$ & $2.21 b c$ & $5.5 \mathrm{c}-\mathrm{f}$ & $4.8 \mathrm{~cd}$ & $4.5 \mathrm{cde}$ & $55.0 \mathrm{bc}$ & $64.9 \mathrm{a}-\mathrm{e}$ & $57.4 \mathrm{cde}$ \\
\hline TMS 95/0211 & $2.27 \mathrm{a}-\mathrm{d}$ & $2.41 \mathrm{bcd}$ & $2.73 a b c$ & $6.2 b-f$ & $6.2 \mathrm{a}-\mathrm{d}$ & $6.0 \mathrm{a}-\mathrm{d}$ & $62.0 \mathrm{abc}$ & $66.2 \mathrm{c}-\mathrm{e}$ & 68.1a-d \\
\hline TME 1 & $2.27 \mathrm{a}-\mathrm{d}$ & $2.93 a-d$ & $3.13 a b c$ & 7.2a-d & $7.2 \mathrm{abc}$ & $7.6 \mathrm{ab}$ & $59.4 \mathrm{abc}$ & $67.7 \mathrm{a}-\mathrm{e}$ & $56.9 \mathrm{de}$ \\
\hline TMEB 693 & $1.97 \mathrm{~cd}$ & $2.35 \mathrm{bcd}$ & $2.53 \mathrm{a}$ & $8.0 \mathrm{a}-\mathrm{c}$ & $8.5 \mathrm{a}$ & $7.7 \mathrm{ab}$ & $52.2 \mathrm{bc}$ & $57.4 \mathrm{e}$ & $57.7 \mathrm{~b}-\mathrm{e}$ \\
\hline TMS 01/0046 & $1.94 \mathrm{~cd}$ & $2.22 \mathrm{~cd}$ & $2.20 \mathrm{bc}$ & 7.0a-e & $6.8 \mathrm{a}-\mathrm{d}$ & $6.3 a b c$ & $61.1 \mathrm{abc}$ & 66.6a-e & $69.3 \mathrm{a}-\mathrm{d}$ \\
\hline TMS 01/0093 & $2.80 \mathrm{a}$ & $3.78 \mathrm{a}$ & $3.66 \mathrm{a}$ & $8.2 \mathrm{ab}$ & $8.8 \mathrm{a}$ & $6.7 a b c$ & $55.4 \mathrm{bc}$ & $58.5 \mathrm{e}$ & $66.2 \mathrm{a}-\mathrm{e}$ \\
\hline TMS 00/0338 & $2.49 \mathrm{abc}$ & $2.74 \mathrm{bcd}$ & $2.85 \mathrm{abc}$ & 7.3a-d & $6.5 \mathrm{a}-\mathrm{d}$ & $5.7 \mathrm{a}-\mathrm{e}$ & $53.9 b c$ & 63.0b-e & 62.6a-e \\
\hline TMS 01/1097 & $2.09 \mathrm{bcd}$ & $2.43 \mathrm{bcd}$ & $2.90 \mathrm{abc}$ & $6.3 \mathrm{a}-\mathrm{f}$ & $6.3 \mathrm{a}-\mathrm{d}$ & $6.0 \mathrm{a}-\mathrm{d}$ & $56.2 \mathrm{abc}$ & $74.0 \mathrm{ab}$ & $63.8 \mathrm{a}-\mathrm{e}$ \\
\hline TMS 01/1086 & $2.11 \mathrm{bcd}$ & $2.79 \mathrm{bcd}$ & $2.94 a b c$ & 5.0def & $6.3 a-d$ & $4.7 \mathrm{cde}$ & $55.0 \mathrm{bc}$ & $61.1 \mathrm{c}-\mathrm{e}$ & $61.3 \mathrm{a}-\mathrm{e}$ \\
\hline TME B 419 & $2.41 \mathrm{abc}$ & $2.54 \mathrm{bcd}$ & $2.78 \mathrm{abc}$ & $4.2 \mathrm{f}$ & $5.1 \mathrm{bcd}$ & $3.2 \mathrm{e}$ & $56.9 \mathrm{abc}$ & $74.1 \mathrm{ab}$ & $72.6 a b$ \\
\hline TMS 30572 & $2.26 \mathrm{a}-\mathrm{d}$ & $2.62 \mathrm{bcd}$ & $3.07 a b c$ & $8.7 \mathrm{a}$ & $8.3 \mathrm{ab}$ & $8.3 \mathrm{a}$ & $60.2 \mathrm{abc}$ & 71.2a-d & 63.7a-e \\
\hline TMS 01/1371 & $2.43 a b c$ & $3.10 \mathrm{abc}$ & $3.16 \mathrm{ab}$ & $5.8 b-f$ & $7.5 a b c$ & $6.5 \mathrm{abc}$ & $53.5 b c$ & $68.5 \mathrm{a}-\mathrm{e}$ & $68.4 a-d$ \\
\hline TMS 01/0085 & $2.23 \mathrm{a}-\mathrm{d}$ & $2.77 \mathrm{bcd}$ & $3.00 \mathrm{abc}$ & $5.8 b-f$ & 7.7abc & $5.5 b-e$ & $58.8 \mathrm{abc}$ & $72.5 \mathrm{abc}$ & 63.3a-e \\
\hline TMS 98/0510 & $2.25 \mathrm{a}-\mathrm{d}$ & $2.79 \mathrm{bcd}$ & $2.98 \mathrm{abc}$ & 7.0a-e & $6.7 \mathrm{a}-\mathrm{d}$ & $5.7 \mathrm{a}-\mathrm{e}$ & $68.3 \mathrm{a}$ & $74.9 \mathrm{a}$ & $74.3 \mathrm{a}$ \\
\hline TMS 01/0131 & $1.94 \mathrm{~cd}$ & $2.34 \mathrm{bcd}$ & $2.23 b c$ & $5.2 \mathrm{def}$ & $5.0 \mathrm{~b}-\mathrm{d}$ & $4.3 \mathrm{cde}$ & $55.4 \mathrm{bc}$ & 67.6a-e & $60.7 \mathrm{~b}-\mathrm{e}$ \\
\hline TMS 98/0505 & $2.05 \mathrm{bcd}$ & $2.32 \mathrm{bcd}$ & $2.12 \mathrm{c}$ & 4.7ef & $3.7 \mathrm{~d}$ & $3.3 \mathrm{de}$ & $61.1 \mathrm{abc}$ & 71.4a-d & $65.2 \mathrm{a}-\mathrm{e}$ \\
\hline TMS 92/0326 & $2.41 \mathrm{abc}$ & $3.02 \mathrm{a}-\mathrm{d}$ & $3.40 \mathrm{a}$ & 7.3a-d & $7.5 \mathrm{a}-\mathrm{c}$ & $6.0 \mathrm{a}-\mathrm{d}$ & $63.7 \mathrm{abc}$ & 72.1a-d & 71.6abc \\
\hline TMS 01/0098 & $2.48 \mathrm{abc}$ & $3.26 \mathrm{ab}$ & $3.43 \mathrm{a}$ & $5.8 b-f$ & $6.8 \mathrm{a}-\mathrm{d}$ & $5.3 b-e$ & $56.8 \mathrm{abc}$ & $62.0 \mathrm{c}-\mathrm{e}$ & $53.9 \mathrm{e}$ \\
\hline TMS 01/1368 & $2.35 \mathrm{abc}$ & $2.53 \mathrm{bcd}$ & $3.09 \mathrm{abc}$ & $4.7 \mathrm{ef}$ & $5.7 \mathrm{a}-\mathrm{d}$ & $5.3 \mathrm{~b}-\mathrm{e}$ & $56.0 \mathrm{abc}$ & $67.7 \mathrm{a}-\mathrm{e}$ & $68.8 \mathrm{a}-\mathrm{d}$ \\
\hline TMS 97/JW2 & $2.77 \mathrm{a}$ & $3.12 \mathrm{ab}$ & $3.52 \mathrm{a}$ & 4.7ef & $6.5 \mathrm{a}-\mathrm{d}$ & $5.3 \mathrm{~b}-\mathrm{e}$ & $51.6 \mathrm{c}$ & $60.4 \mathrm{de}$ & $60.9 \mathrm{a}-\mathrm{e}$ \\
\hline SE & 0.65 & 0.96 & 1.02 & 2.5 & 3.4 & 2.8 & 12.5 & 11.9 & 13.8 \\
\hline $\mathrm{CS} \times \mathrm{V}$ & NS & NS & NS & NS & NS & NS & NS & NS & $\mathrm{S}$ \\
\hline
\end{tabular}

NS $=$ not significant, $\mathrm{S}=$ significant

Table 4: Influence of intercropping on root rot and tuber yield of elite cassava varieties at different age of plant in 2012/2014 cropping season

\begin{tabular}{|c|c|c|c|c|c|c|c|c|c|c|}
\hline \multirow[b]{2}{*}{ Treatment } & \multicolumn{2}{|c|}{ Stem girth (mm) } & \multirow[b]{2}{*}{18 MAP } & \multicolumn{3}{|c|}{ Root rot incidence (\%) } & \multicolumn{4}{|c|}{ Fresh tuber mass $\left(\mathrm{t} \mathrm{ha}^{-1}\right)$} \\
\hline & $12 \mathrm{MAP}$ & 15 MAP & & $\begin{array}{l}12 \\
\text { MAP }\end{array}$ & $\begin{array}{l}15 \\
\text { MAP }\end{array}$ & $\begin{array}{l}18 \\
\text { MAP }\end{array}$ & LER & $\begin{array}{l}12 \\
\text { MAP }\end{array}$ & $15 \mathrm{MAP}$ & $18 \mathrm{MAP}$ \\
\hline \multicolumn{11}{|l|}{ Cropping systems (CS) } \\
\hline Sole & 23.9 & 23.1 & 23.3 & 0.29 & 0.48 & 11.61 & - & 23.7 & 31.0 & 27.0 \\
\hline Intercrop & 23.52 & 23.7 & 23.0 & 0.31 & 0.73 & 11.06 & - & 24.6 & 31.5 & 26.6 \\
\hline LSD & NS & NS & NS & NS & NS & NS & - & NS & NS & NS \\
\hline \multicolumn{11}{|l|}{ Variety (V) } \\
\hline TMS 98/0581 & $27.8 \mathrm{ab}$ & $24.7 \mathrm{abc}$ & $24.4 \mathrm{a}$ & 0.39 & 1.60 & 35.14 & 1.46 & $29.3 \mathrm{ab}$ & 30.0 & 23.6 \\
\hline TMS 01/1797 & $22.9 \mathrm{c}-\mathrm{g}$ & $21.2 \mathrm{bcd}$ & $23.1 \mathrm{ab}$ & 0.14 & 0.44 & 4.93 & 1.23 & $22.1 \mathrm{a}-\mathrm{d}$ & 29.2 & 26.7 \\
\hline TMS 95/0211 & $23.8 b-f$ & $24.9 \mathrm{abc}$ & $24.1 \mathrm{a}$ & 0.5 & 2.39 & 10.99 & 1.10 & $24.5 \mathrm{abc}$ & 38.9 & 35.8 \\
\hline TME 1 & $23.3 \mathrm{c}-\mathrm{g}$ & $25.0 \mathrm{abc}$ & 23.0ab & 0.12 & 0.94 & 28.02 & 1.46 & $26.7 \mathrm{abc}$ & 26.5 & 22.4 \\
\hline TMEB 693 & $22.1 \mathrm{~d}-\mathrm{g}$ & $22.2 \mathrm{a}-\mathrm{d}$ & $20.7 \mathrm{ab}$ & 0.0 & 0.50 & 14.40 & 1.06 & $19.7 \mathrm{cde}$ & 25.4 & 27.2 \\
\hline TMS 01/0046 & $25.2 \mathrm{a}-\mathrm{e}$ & $20.7 \mathrm{~cd}$ & $21.0 \mathrm{ab}$ & 0.94 & 1.40 & 11.20 & 1.36 & $27.4 \mathrm{abc}$ & 37.3 & 25.5 \\
\hline TMS 01/0093 & $20.8 f-g$ & $22.2 \mathrm{a}-\mathrm{d}$ & $22.2 \mathrm{ab}$ & 0.24 & 0.56 & 12.10 & 1.10 & $22.6 a-d$ & 30.7 & 26.2 \\
\hline TMS 00/0338 & $29.2 \mathrm{a}$ & $22.7 \mathrm{a}-\mathrm{d}$ & $27.1 \mathrm{a}$ & 0.73 & 0.73 & 7.57 & 1.06 & $32.3 \mathrm{a}$ & 29.1 & 29.5 \\
\hline TMS 01/1097 & $24.7 b-f$ & $25.1 \mathrm{abc}$ & $23.3 \mathrm{ab}$ & 0.24 & 1.43 & 5.36 & 1.23 & $29.6 \mathrm{ab}$ & 31.7 & 25.8 \\
\hline TMS 01/1086 & $21.9 \mathrm{efg}$ & $21.6 a-6$ & $21.6 \mathrm{ab}$ & 0.00 & 0.78 & 17.48 & 1.46 & $23.1 \mathrm{a}-\mathrm{d}$ & 29.2 & 24.4 \\
\hline TME B 419 & $23.0 \mathrm{c}-\mathrm{g}$ & $24.7 \mathrm{abc}$ & $24.4 \mathrm{a}$ & 0.27 & 0.80 & 4.74 & 1.23 & 21.3a-d & 27.0 & 20.8 \\
\hline TMS 30572 & $23.7 b-f$ & $22.5 a-d$ & $25.3 \mathrm{a}$ & 0.14 & 2.09 & 21.97 & 1.23 & $32.0 \mathrm{a}$ & 39.0 & 35.4 \\
\hline TMS 01/1371 & $24.2 b-f$ & $26.3 \mathrm{a}$ & $24.4 \mathrm{a}$ & 0.00 & 1.78 & 20.02 & 1.06 & $25.0 \mathrm{abc}$ & 37.5 & 32.7 \\
\hline TMS 01/0085 & $22.8 \mathrm{c}-\mathrm{g}$ & $23.1 \mathrm{a}-\mathrm{d}$ & $23.3 \mathrm{ab}$ & 0.56 & 0.65 & 4.61 & 1.10 & $25.6 \mathrm{abc}$ & 33.6 & 30.5 \\
\hline TMS 98/0510 & $26.5 \mathrm{abc}$ & $23.5 \mathrm{a}-\mathrm{d}$ & $26.2 \mathrm{a}$ & 0.27 & 1.24 & 18.34 & 1.53 & $26.6 \mathrm{abc}$ & 30.4 & 24.7 \\
\hline TMS 01/0131 & $22.2 \mathrm{~d}-\mathrm{g}$ & $23.7 \mathrm{a}-\mathrm{d}$ & $21.8 \mathrm{ab}$ & 0.27 & 1.49 & 4.25 & 1.06 & $20.1 \mathrm{a}-\mathrm{d}$ & 24.2 & 20.2 \\
\hline TMS 98/0505 & $19.5 \mathrm{~g}$ & $22.0 \mathrm{a}-\mathrm{d}$ & $17.7 \mathrm{~b}$ & 0.14 & 1.01 & 11.33 & 1.20 & $15.2 \mathrm{~cd}$ & 23.1 & 17.7 \\
\hline TMS 92/0326 & $23.7 b-f$ & $26.2 \mathrm{ab}$ & $23.7 \mathrm{a}$ & 0.00 & 1.43 & 16.31 & 1.10 & $30.1 \mathrm{ab}$ & 38.5 & 31.8 \\
\hline TMS 01/0098 & $24.0 \mathrm{~b}-\mathrm{f}$ & $24.4 \mathrm{a}-\mathrm{d}$ & 23.0ab & 0.71 & 1.01 & 2.85 & 1.40 & $22.0 \mathrm{a}-\mathrm{d}$ & 33.3 & 32.3 \\
\hline TMS 01/1368 & $26.2 \mathrm{a}-\mathrm{d}$ & $25.0 \mathrm{abc}$ & $23.8 \mathrm{a}$ & 0.27 & 0.56 & 2.92 & 1.30 & $21.6 a-d$ & 39.4 & 21.2 \\
\hline TMS 97/JW2 & 21.0fg & $19.5 d$ & $21.4 \mathrm{ab}$ & 0.94 & 0.27 & 8.80 & 1.50 & $12.0 \mathrm{~d}$ & 21.5 & 28.3 \\
\hline SE & 4.2 & 5.0 & 5.8 & NS & NS & NS & NS & 12.3 & NS & NS \\
\hline CS X V & NS & NS & $\mathrm{NS}$ & NS & NS & NS & NS & NS & NS & NS \\
\hline
\end{tabular}

$\mathrm{NS}=$ not significant, $\mathrm{S}=$ significant 


\subsection{Influence of age at harvest and intercropping on the plant height, tuber girth and tuber rot of elites cassava varieties at 12, 15 and 18 MAP}

The pooled mean plant height, tuber girth and tuber rot obtained from 2012/2014 showed significant difference among the ages of plant at harvest (Table 5). The similarity in height and tuber girth of plants harvested at 15 and 18 MAP buttressed the fact that maturity had been attained compared to 12 MAP. The higher incidence root rot recorded in 18 MAP accounted for the apparent decline in fresh tuber mass. This result contradicts the finding of Mulualem and Ayenew (2012) who recommended 18 months as the appropriate age to harvest cassava to get the desired yield.

The pool mean of varieties TMS 01/0093, 97/JW2 and $01 / 0098$ had similar but the tallest plants. However, varieties 97/JW2 and 01/0098 were not significantly taller than those of TMS 92/0326, TMS 98/0581 and TMS 01/1371. The following varieties TMS 01/0131, TMS 98/0505, TMS 01/1797, TMEB 693 and TMS $01 / 0046$ had the shortest plants. The pooled mean fresh tuber number of varieties TMS 30572, TMEB 693, TMS 01/0093 and TME 1 were similar and highest among the others. But the tuber number of varieties TMS 01/0093 and TME 1 were not significantly higher than at TMS 92/0326. Variety TMS 98/0505 had the minimum number of fresh tubers but not significantly lower than TMS 01/1797, TME B 419 and TMS 01/0131.The tuber girth of varieties TMEB 693, TMS 01/0093, TMS 00/0338, TMS 01/1086, TMS 97/JW2, TMS 01/1797 and TMS 01/0098 were similar but significantly lower than those of TMS 98/0510, TMS 92/0326, TME B 419 and TMS 98/0581. The stem girth of varieties TMS 98/0510, TMS 00/0338 and TMS 98/0581 were significantly higher compared to others whereas TMS 97/JW2 and TMS 98/0505 had the lowest.

\subsection{Influence of age at harvest and intercropping on the tuber yield of elites cassava varieties at 12, 15 and 18 MAP}

Although the tuber yield recorded for the three ages were similar but dropped at 18 MAP evidently due to rot damage. Ebah-Djedji et al. (2012) reported decline in cassava tuber at 17 months old, however, the decline was not linked to root rot. Hammer et al. (1987) reported that root rot occurred in the second year but was not specific on the number of months.

The tuber fresh mass of varieties were significantly different from one another. The following varieties TMS 30572, TMS 92/0326, TMS 95/0211, TMS 01/1371, TMS 00/0338, TMS 01/0046, TMS 00/0098 and TMS 01/1097 were among the topmost eight varieties whereas TMEB693, TMEB 419, 01/0131, TMS 97/JW2 and TMS 98/0505 were the least.

Generally, the consistence in plant heights values at 12 MAP of varieties TMS 97/JW2, TME B 419 and TMS 01/1371 (which ranked among the top) and those of TMS 01/0131, TMS 01/0046 and TMS 98/0505 (at the bottom of the list) in both cropping seasons are indication of their stability despite differences in crop management. Besides, the ability of TMS 30572, TMS 92/0326 and TMS 98/0581 to constantly rank among the first six varieties at 12 MAP in the two seasons makes them candidates to be recommended to farmers (Table 6). The wide gap in tuber yield between the two cropping seasons could be attributed to the fertilizer application. Although, farmers hardly use fertilizer for cassava production because of the notion that cassava can thrive on marginal soils that cannot sustain other crops. This trial connotes that the addition of fertilizer can substantially enhance tuber yield. Odedina et al. (2012) who worked on integrated nutrient management reported similar gap between control and other sources of nutrient. Ironically, appreciable quantity of root rot was observed in the first cropping season compared to the second, it was not quite clear if the addition of fertilizer was responsible for the tuber rot. Consequently, there is the need to validate whether or not fertilizer application to cassava influences root rot.

\section{CONCLUSIONS}

The study has shown that the cassava varieties were not affected by intercropping but Land Equivalent Ratio was above one in both cropping seasons indicating that the performance of the improved varieties in intercrop was efficient. Plant height and tuber girth were higher in 15 and 18 MAP than 12. On the bases of their consistent performance at $12 \mathrm{MAP}$, in the two cropping seasons, TMS 30572, TMS 92/0326 and TMS 98/0581 are candidate varieties to be recommended to farmer with or without resource constraints. The pooled mean tuber yield showed that TMS 30572, TMS 92/0326, TMS 95/0211, TMS 01/1371, TMS 00/0338, TMS 01/0046, TMS 00/0098, TMS 01/1097, TMS 01/0085, TMS 98/0581 and TMS 98/510 are top eight varieties. The incidence of tuber rot was highest at 18 MAP hence; harvesting could be delayed up to 15 MAP to reduce 


\section{J. N. ODEDINA et al.}

tuber rot. The three yellow flesh tuber varieties identified had comparable performance with their white

counterpart.

Table 5: Performance of elite cassava varieties as influenced by age of plant at harvest and intercropping in 2012/2014 cropping season

\begin{tabular}{|c|c|c|c|c|c|c|}
\hline Treatment & $\begin{array}{l}\text { Plant height } \\
(\mathrm{m})\end{array}$ & $\begin{array}{l}\text { Fresh tuber } \\
\text { no.plant }^{-1}\end{array}$ & $\begin{array}{l}\text { Tuber girth } \\
(\mathrm{mm})\end{array}$ & $\begin{array}{l}\text { Stem girth } \\
(\mathrm{mm})\end{array}$ & $\begin{array}{l}\text { Root rot incidence } \\
(\%)\end{array}$ & $\begin{array}{l}\text { Fresh tuber mass } \\
\left(\mathrm{t} \mathrm{ha}^{-1}\right)\end{array}$ \\
\hline \multicolumn{7}{|c|}{ Age at harvest $(\mathrm{H})$} \\
\hline $12 \mathrm{MAP}$ & $2.28 \mathrm{~b}$ & 6.2 & $57.9 \mathrm{~b}$ & 23.73 & $1.95 \mathrm{~b}$ & 24.12 \\
\hline 15 MAP & $2.73 \mathrm{a}$ & 6.6 & $67.0 \mathrm{a}$ & 23.39 & $0.90 \mathrm{c}$ & 31.23 \\
\hline 18 MAP & $2.90 \mathrm{a}$ & 5.7 & $64.6 \mathrm{a}$ & 23.17 & $3.43 \mathrm{a}$ & 26.78 \\
\hline LSD & 0.30 & NS & 5.23 & NS & 0.67 & NS \\
\hline \multicolumn{7}{|c|}{ Cropping systems (CS) } \\
\hline Sole & $2.61 \mathrm{a}$ & $6.1 \mathrm{a}$ & $62.8 \mathrm{a}$ & $23.44 \mathrm{a}$ & $2.09 \mathrm{a}$ & $27.21 \mathrm{a}$ \\
\hline Intercrop & $2.67 \mathrm{a}$ & $6.2 \mathrm{a}$ & $63.5 \mathrm{a}$ & $23.42 \mathrm{a}$ & $2.09 \mathrm{a}$ & $27.55 \mathrm{a}$ \\
\hline LSD & NS & NS & NS & NS & NS & NS \\
\hline $\mathrm{H} \times \mathrm{CS}$ & NS & NS & NS & NS & NS & NS \\
\hline \multicolumn{7}{|l|}{ Variety (V) } \\
\hline TMS 98/0581 & $2.95 \mathrm{bcd}$ & 6.1defg & $66.6 b c$ & $25.6 \mathrm{ab}$ & $2.89 \mathrm{a}$ & 27.76bcdefg \\
\hline TMS 01/1797 & $2.01 \mathrm{j}$ & 4.9hijk & $59.1 \mathrm{fgh}$ & $22.4 \mathrm{fgh}$ & $1.52 \mathrm{a}$ & 26.01defgh \\
\hline TMS 95/0211 & 2.47fghi & 6.1defg & $65.5 \mathrm{bcd}$ & $24.2 \mathrm{bcdef}$ & $2.33 \mathrm{a}$ & $32.98 \mathrm{abc}$ \\
\hline TME 1 & $2.78 \mathrm{cdef}$ & $7.3 \mathrm{abc}$ & 61.4defg & $23.8 \mathrm{bcdef}$ & $2.49 \mathrm{a}$ & 25.21defgh \\
\hline TMEB 693 & $2.28 \mathrm{ghij}$ & $8.1 \mathrm{a}$ & $55.8 \mathrm{~h}$ & $21.7 \mathrm{gh}$ & $1.76 \mathrm{a}$ & 24.10efghi \\
\hline TMS 01/0046 & $2.12 \mathrm{ij}$ & $6.7 \mathrm{~cd}$ & $65.7 \mathrm{bcd}$ & 22.6efg & $2.71 \mathrm{a}$ & 30.09abcde \\
\hline TMS 01/0093 & $3.41 \mathrm{a}$ & 7.9ab & 60.0efgh & $21.7 \mathrm{gh}$ & $1.99 \mathrm{a}$ & $26.53 \mathrm{cdefgh}$ \\
\hline TMS 00/0338 & $2.69 \mathrm{def}$ & $6.5 \mathrm{cde}$ & 59.8efgh & $26.4 \mathrm{a}$ & $2.25 \mathrm{a}$ & 30.31abcde \\
\hline TMS 01/1097 & $2.48 \mathrm{fgh}$ & $6.2 \mathrm{cdefg}$ & $64.3 \mathrm{bcde}$ & $24.4 \mathrm{bcde}$ & $1.61 \mathrm{a}$ & 29.04abcdef \\
\hline TMS 01/1086 & 2.61defg & 5.3fghi & $59.2 \mathrm{fgh}$ & $21.7 \mathrm{gh}$ & $1.89 \mathrm{a}$ & $25.57 \mathrm{defgh}$ \\
\hline TME B 419 & $2.58 \mathrm{efg}$ & $4.1 \mathrm{jk}$ & 67.9abc & $24.0 \mathrm{bcdef}$ & $1.77 \mathrm{a}$ & $23.04 \mathrm{fghi}$ \\
\hline TMS 30572 & $2.65 \mathrm{def}$ & $8.4 \mathrm{a}$ & $65.1 \mathrm{bcd}$ & $23.8 \mathrm{bcdef}$ & $2.27 \mathrm{a}$ & $35.48 \mathrm{a}$ \\
\hline TMS 01/1371 & $2.90 \mathrm{~b}-\mathrm{e}$ & $6.6 \mathrm{cde}$ & $63.5 \mathrm{cdef}$ & 25.0abcd & $1.98 \mathrm{a}$ & 31.72abcd \\
\hline TMS 01/0085 & $2.67 \mathrm{def}$ & $6.3 \mathrm{cdefg}$ & $64.9 \mathrm{bcd}$ & 23.1defg & $2.09 \mathrm{a}$ & $28.88 \mathrm{bcdef}$ \\
\hline TMS 98/0510 & $2.67 \mathrm{def}$ & $6.4 \mathrm{cdefg}$ & $72.5 \mathrm{a}$ & $25.4 \mathrm{ab}$ & $2.37 \mathrm{a}$ & 27.21bcdefg \\
\hline TMS 01/0131 & $2.17 \mathrm{hij}$ & $4.8 \mathrm{ijk}$ & $61.2 \mathrm{defg}$ & 22.6efg & $1.64 \mathrm{a}$ & $21.52 \mathrm{ghi}$ \\
\hline TMS 98/0505 & 2.16hij & $3.9 \mathrm{k}$ & $65.9 \mathrm{bcd}$ & $19.7 \mathrm{i}$ & $1.93 \mathrm{a}$ & $18.64 \mathrm{i}$ \\
\hline TMS 92/0326 & $2.94 \mathrm{bcd}$ & $6.9 \mathrm{bcd}$ & 69.1ab & 24.5abcde & $2.02 \mathrm{a}$ & $33.68 \mathrm{ab}$ \\
\hline TMS 01/0098 & $3.06 \mathrm{abc}$ & 6.0defgh & $57.5 \mathrm{gh}$ & $23.8 \mathrm{bcdef}$ & $2.00 \mathrm{a}$ & 29.19abcdef \\
\hline TMS 01/1368 & $2.65 \mathrm{def}$ & $5.2 \mathrm{ghij}$ & $64.2 \mathrm{cde}$ & $25.0 \mathrm{abcd}$ & $1.66 \mathrm{a}$ & 27.40bcdefg \\
\hline TMS 97/JW2 & $3.15 \mathrm{ab}$ & 5.5efghi & $57.6 \mathrm{gh}$ & $20.6 \mathrm{hi}$ & $2.74 \mathrm{a}$ & $20.58 \mathrm{hi}$ \\
\hline $\mathrm{SE}(\mathrm{V})$ & 0.36 & $1.18^{\circ}$ & 4.9 & 2.0 & NS & 6.56 \\
\hline HX V & NS & NS & NS & NS & NS & NS \\
\hline $\mathrm{CS} \times \mathrm{V}$ & NS & NS & NS & NS & $\mathrm{S}$ & NS \\
\hline $\mathrm{H} \times \mathrm{CS}$ & NS & NS & NS & NS & NS & NS \\
\hline $\mathrm{H} \times \mathrm{CS} \times \mathrm{V}$ & NS & NS & NS & NS & NS & NS \\
\hline
\end{tabular}

$\mathrm{NS}=$ not significant, $\mathrm{S}=$ significant

\section{ACKNOWLEDGEMENTS}

The authors wish to thank the Institute of Food Security, Environmental Resources and Agricultural Research (IFSERAR), Federal University of Agriculture, Abeokuta for funding the project and Institute
International of Tropical Agriculture (IITA), Ibadan for providing the bulky cassava planting materials and technical support in data collection.

\section{REFERENCES}

Akparobi, S.O., Togun S.O. and Ekanayake, I.J. (1998). Assessment of cassava genotypes for resistance to cassava mosaic disease, cassava bacterial blight and cassava green mite at lowland and mid altitude site in Nigeria. African. Crop Science. Journal, 6, 385396. doi:10.4314/acsj.v6i4.27790
Benesi, I.R.M., Labuschagne, M.T., Herselman, L. Mahungu, N.M. and Saka, J.K. (2008).The effect of genotype, location and season on cassava starch extraction. Euphytica, 160, 59-74. doi:10.1007/s10681-007-9589-x 
Ebah-Djedji, B.C., Dje, K.M., N'Zue, B., Zohouri, G.P. and Amani, N.G. (2012). Effect of harvest period on starch and dry matter content from the tuberous roots of improved cassava (Manihot esculenta Crantz) varieties. Pakistan Journal of nutrition, 11 (5), 414-418 doi:10.3923/pjn.2012.414.418

Ekanayake, I.J., Osiru, D.S.O. and Porto, M.C.M. (1997). Agronomy of cassava IITA Research Guide, $60 \mathrm{pp} 1-20$

Food and Agriculture Organization Statistics (FAOSTAT) (2003). www.faostat. Org. Accessed $1 / 04 / 2013$

FAOSTAT. (2010). www.faostat. Org Accessed $10 / 03 / 2013$

FAO (2005) FAO STATS. Food and Agriculture Organization of United Nations, Rome, Italy. http://www.fao.org. Accessed 07/05/2012

FAO

http://fao.org/faostat/en/\#rankings/commodities_by _countries. Commodities by Countries search. Accessed by $1 / 08 / 2017$

FAO

http://fao.org/faostat/en/\#rankings/countries_by_co mmodities. Countries by Commodities search. Accessed by $1 / 08 / 2017$

Hammer, G. L., Hobman, F. R. and Shepherd, R. K. (1987). Effects of Planting Time and Harvest Age on Cassava (Manihot esculenta) in Northern Australia. I. Crop Growth and Yield in Moist Environments. Experimental Agriculture, ,23, 401414. doi:10.1017/S0014479700017361

Howeler, R.H. (2007). Agronomic practices for sustainable cassava production in Asia. In: Centro Internacional de Agricultura Tropical (CIAT). Cassava research and development in Asia: Exploring new opportunities for an ancient crop. Proceedings of the $7^{\text {th }}$ Regional Workshop, held in Bangkok, Thailand, Oct 28 - Nov 1.

International Institute of Tropical Agriculture (IITA) (1987). Annual Report of Root, Tuber and Plantain Improvement Program. Ibadan, Nigeria. 90 pp. IITA

Institute of Food Security, Environmental Resources and Agricultural Research, (IFSERAR) Federal University of Agriculture, Abeokuta FUNAAB. (2009). Diagnostic survey of Southwestern Agricultural zone of Nigeria. www.unaab.edu.ng. Accessed 10/01/2015.

Kogram, C., Maneekao, S., Poosri Boonguer. (2002). Influence of chicken manure on cassava yield and soil properties. Paper presented at $17^{\text {th }}$ WCSS 1421 August 2002, Bangkok, Thailand
Lebot, V. (2009). Tropical roots and tuber crops: cassava, sweet potato, yams and aroids. Crop Production science in horticulture series. 17, CABI Publishing, Londres, pp. 50-103.

Maroya, N. G., Asante, I. K. and Dixon, A. (2010). Genotype by environment interaction effect on beta-carotene of yellow root cassava (Manihot esculenta Crantz) genotypes in Ghana. Proc. 11th ISTRC-AB Symposium. Kinshasa, DR Congo. 4-8 October, 2010.

Mulualem, T. and Ayenew, B. 2012. Cassava (Manihot esculentus Cranz) varieties and harvesting stages influenced yield and yield related component. Journal of Natural Science Research, 2, (10), 122128.

Mwangi, M., Bandyopadhyay, R., Dixon, A.G.O. and Tatahangy, W. (2004). The status of fungal rot diseases as constraint to cassava production and utilization in eastern Democratic Republic of Congo. Proceedings of $9^{\text {th }}$ Triennial Symposium of the International Society of Tropical Root CropsAfrica Branch, held from 30st October $-5^{\text {th }}$ November, at Whitesands Hotel, Mombasa, Kenya.

Odedina, J.N. Ojeniyi, S. and Odedina, S.A. (2012). Integrated nutrient management for sustainable cassava production in south Western Nigeria. Archives of Agronomy and Soil Science, 58 (1), 132 - 140. doi:10.1080/03650340.2012.695865

Odedina, J.N., Ojeniyi, S., Odedina, S.A., Fabunmi, T.O., Olowe, V. (2015). Growth and yield responses of cassava poultry manure and time of harvest in rainforest Agro-ecological zone of Nigeria. International Journal of Agricultural Sciences and Natural Resources, 2, (3), 67-72

Oyeka, T.J. (2004). Cassava root rot fungi in Nigeria; variability in Botryodiplodia theobromae isolates and evaluation of cassava germplasm for root rot resistance. $\mathrm{PhD}$ thesis University of Ibadan .

Sagrilo, E. Filho, P.S.V. Pequeno, M.G.,Vidigal,' M.C.G., Scapim, C. A., Kvitschal, M.V., Maia, R.R. and Rimoldi, F. (2006). Effect of harvest period on foliage Production and dry matter distribution in five cassava cultivars during the second plant cycle. Brazilian Archives of Biology and Technology, 49,(6), 1007-1018. doi:10.1590/S1516-89132006000700019

Ssemakula Gorrettie and Alfred Dixon (2007). Genotype X environment interaction, stability and agronomic performance of carotenoid-rich cassava clones. Scientific Research and Essay, 2 (9), 390399. 